\title{
ATENÇÃO FARMACÊUTICA NO USO DE BENZODIAZEPÍNICOS E OUTROS PSICOFÁRMACOS NO TRATAMENTO DE TRANSTORNOS DE ANSIEDADE E PÂNICO POR JOVENS ATUALMENTE NO MUNICÍPIO DE NOVA IGUAÇU
}

\author{
Igor Brandão Lisboa ${ }^{1}$ \\ Luciana Ferreira Mattos Colli²
}

RESUMO: Introdução: Os Transtornos de ansiedade têm sido cada vez mais diagnosticados em indivíduos cada vez mais jovens, até mesmo em crianças essa doença vem sendo observada com o passar dos anos. Além disso, o fato de ser uma doença com possivelmente mais de roo sintomas diferentes torna ainda mais delicado o tratamento para este problema. Metodologia: $O$ tratamento farmacológico deve ser devidamente esclarecido, inclusive o tempo de que o medicamento precisa para que o paciente comece a perceber os efeitos, isso pode evitar uma possível não adesão ao tratamento e ainda possíveis intoxicações por interação com outras substâncias. Objetivo: Este estudo tem o objetivo de demonstrar a eficiência dos fármacos nos pacientes que sofrem com transtornos de ansiedade e alternativas aos pacientes que não necessitam desse tipo de medicação de acordo com os determinados sintomas, com base em banco de dados e literaturas. Resultados: Percebe-se que os benzodiazepínicos têm uma melhor eficácia em relação à transtornos do pânico e seus sintomas mais físicos, enquanto os inibidores de recaptação de serotonina tem uma melhora nos sintomas psicológicos da ansiedade sendo também uma alternativa mais branda e com menos efeitos colaterais. Conclusão: Há pouca informação no momento da consulta, falta de esclarecimento sobre efeitos inerentes aos medicamentos que levam à tolerância e potencialização da cronicidade.

Palavras chaves: Transtorno de Ansiedade. Psicotrópicos. Jovens. Atenção Farmacêutica.

ABSTRACT: Introduction: Anxiety disorders have been increasingly diagnosed in younger people, even in children, this disease has been observed over the years. Furthermore, the fact that it is a disease with possibly more than a hundred different symptoms makes the treatment for this problem even more delicate. Methodology: The pharmacological treatment must be properly explained, including the time it takes the drug for the patient to start noticing the effects, this can avoid a possible non-adherence

${ }^{\text {I }}$ Gradução pela Universidade Iguaçu.

${ }^{2}$ Orientadora. Universidade Iguaçu 
to the treatment. Objective: This study aims to demonstrate the effectiveness of drugs to patients who care for anxiety disorders and alternatives to patients who are not this type of medication according to symptoms, based on database and literature. Results: It is noticed that benzodiazepines have a better efficacy in relation to panic disorders and its more physical symptoms, while serotonin reuptake inhibitors have an improvement in the psychological symptoms of anxiety. Conclusion: There is little information at the time of consultation, lack of clarification on the effects inherent to medications that lead to non-adherence to treatment.

Keywords: Anxiety Disorders. Psychotropics. Youth. Pharmaceutical Attention.

\section{INTRODUÇÃO}

Ansiedade pode ser caracterizada por uma sensação forte de medo, agonia, que gera uma tensão e desconforto por antecipação do perigo, ainda que de algo irracional. $O$ sentimento ansiedade por si só não é caracterizado como uma patologia, porém, se torna ao se transformar em algo exagerado, desproporcional e fora de controle em relação ao estímulo, especialmente levando-se em conta quando se observa a norma naquela determinada faixa etária (CARVALHO et al., 2017).

Ao contrário do que se imagina pela maior parte dos indivíduos, os transtornos de pânico e ansiedade não são meramente ilusórios como muitos imaginam que sejam. O TP, assim chamado o transtorno de pânico, consiste em uma sensação de medo e apreensão intensos precedidos por sintomas físicos e cognitivos que tem seu início de forma brusca e repentina, tendo seu platô por volta de dez minutos. Esses ataques levam a preocupações constantes, alterando o comportamento de quem sofre com a patologia, considerando a ocorrência de novos ataques de pânico (SALUM et al., 2009).

A vida cotidiana dos jovens e a velocidade com que as informações são entregues estão consideravelmente maiores do que há anos, isso somado a traumas e situações de convívio, ao sedentarismo e má alimentação pode desencadear alguns transtornos psiquiátricos como é o caso dos transtornos de ansiedade generalizada, síndrome do pânico e até mesmo a depressão, que vem acometendo grande partes dos jovens na 
sociedade contemporânea, onde até Io\% das crianças e adolescentes podem apresentar algum tipo de quadro patológico nesse sentido (CANDIDO et al., 2017).

Os benzodiazepínicos (BZD) estão entre os medicamentos mais vendidos ao redor do mundo devido a sua ação depressora do sistema nervoso central. São utilizados principalmente para tratamento de ansiedade, distúrbios do sono, como miorrelaxantes, como anticonvulsivante e ainda como indutor de anestesia como é o caso do midazolam. (SILVA et al., 2018).

É necessário que seja dada a devida atenção quanto ao nível de dependência causada por medicamentos psicotrópicos, especialmente quando o consumo exagerado ocorre por jovens no início de suas vidas, podendo ser usuários de drogas recreativas e ainda pessoas com distúrbios psiquiátricos ou emocionais em que pensam nesses medicamentos como alternativas ao suicídio (SILVA et al., 2018). Os benzodiazepínicos, como visto anteriormente, possuem um enorme potencial para causar dependência e tolerância, portanto, é de extrema importância a orientação quanto a introdução e retirada do medicamento, devendo ser feita lentamente para que não ocorram crises de abstinência, já que o medicamento pode causar grande tolerância de forma rápida (FERNANDES et al., 2018).

Levando em consideração alguns aspectos majoritários da juventude, pode-se considerar que uma grande parte dos jovens costuma consumir bebidas alcóolicas, entre outras substâncias depressoras e/ou estimulantes do sistema nervoso central, ainda que apenas socialmente. Os efeitos fisiológicos dos benzodiazepínicos, por exemplo, irão potencializar os efeitos em caso de haver outras substâncias depressoras ou ainda, causar outros tipos de problemas inesperados em caso de haver utilização juntamente com estimulantes do SNC (SILVA et al., 2018).

Além disso, é fundamental esclarecer aos jovens os possíveis efeitos inerentes ao medicamento benzodiazepínico como é o caso da sonolência e diminuição dos reflexos. $\mathrm{E}$, ainda, há efeitos causados pelo uso inadequado deles, que levam à diminuição da 
cognição, sedação, tolerância, entre outros. $O$ pior ocorrer ao administrar o medicamento juntamente com o consumo de bebidas alcóolicas (FONTES et al., 2020), muitas vezes pela falta de informação no momento da consulta, pode levar o indivíduo até mesmo a um estado de coma de acordo com a quantidade de ambas as substâncias, ainda que os benzodiazepínicos sejam relativamente mais seguros que seu predecessor, os barbitúricos, que possuem um índice terapêutico consideravelmente menor (DEMENECH et al., 2020).

Este trabalho tem o objetivo de alertar sobre os perigos do consumo de determinadas substâncias em paralelo a outras, demonstrar aspectos clínicos da ansiedade e do transtorno de pânico, indicar alternativas para evitar que haja danos e até mesmo acidentes provenientes da falta de conhecimento sobre o assunto, bem como diagnósticos e avanços no tratamento. É de extrema importância o diálogo no momento do diagnóstico e também na entrega do medicamento e que se realize um apanhado geral sobre a vida do jovem em questão para mensurar a possibilidade de danos em caso de administração incorreta.

\section{I.I Objetivo Geral}

Demonstrar a importância da informação no momento da consulta e possíveis alternativas para medicamentos que podem levar à dependência.

\subsection{Objetivos Específicos}

Demonstrar alguns dos inúmeros sintomas que são desencadeados pelos transtornos de ansiedade e do pânico;

Evidenciar alternativas medicamentosas ou não medicamentosas para ajudar a tratar os sintomas advindos de crises de pânico ou da ansiedade;

Expor os perigos na administração de medicamentos que agem no sistema nervoso central e suas respectivas interações medicamentosas, principalmente quando se trata de automedicação; 
Identificar a importância em se conhecer sobre as condições patológicas da mente humana na atualidade.

\section{METODOLOGIA}

Este estudo tem a pretensão de avaliar artigos e reunir informações presentes em bancos de dados como Scientific Electronic Library Online (Scielo) entre outras, em que foram utilizadas técnicas metodológicas relevantes de 2013 à 2021. A pesquisa leva em conta alguns psicofármacos como antidepressivos, hipnóticos, sedativos, ansiolíticos, porém com ênfase em benzodiazepínicos e a relação dos mesmos com a vida cotidiana dos jovens e seu início com a medicação e possíveis alternativas para os mesmos. Foram utilizados artigos nos idiomas português e inglês.

\section{BENZODIAZEPÍNICOS}

$\mathrm{Na}$ atualidade há uma gigantesca variedade de psicotrópicos que visam melhorar a qualidade de vida dos jovens com transtornos psicológicos, dentre eles temos os benzodiazepínicos, antidepressivos, anticonvulsivantes, barbitúricos, hipnóticos ou sedativos.

O primeiro fármaco benzodiazepínico a ser descoberto foi o Clordiazepóxido pelo laboratório Hoffman-La Roche durante os anos 6o e teve seus efeitos terapêuticos reconhecidos na década de 7o, época em que começou a ser comercializado e tornando-se uma das classes medicamentosas mais prescritas do mundo (SILVA et al., 2018).

O clordiazepóxido teve seus efeitos hipnóticos, miorrelaxantes e ansiolíticos relacionados ao abuso e síndrome de abstinência, levando a dependência e tolerância, o que fez com que o medicamento fosse restrito na década seguinte (SILVA et al., 2018).

Os benzodiazepínicos podem ser classificados com relação à sua meia vida plasmática, podendo ser medicamentos de ação muito curta, curta, intermediária e longa, em que seu tempo de meia vida funciona proporcionalmente à sua ação farmacológica, 
então, pode-se dizer que medicamentos com maior tempo de meia vida, maior será a resposta resultante do acúmulo do fármaco nos tecidos (SILVA et al., 2018).

O mecanismo de ação dos BZD se dá pela ação seletiva sobre os receptores GABAa, o que resulta numa intensificação da resposta do neurotransmissor Ácido Gama-Aminobutírico ou GABA e assim aumentando o influxo de cloreto que leva a uma hiperpolarização da membrana plasmática de células nervosas, diminuindo sua excitabilidade (SILVA et al., 2018).

Figura I - Ligação ao receptor

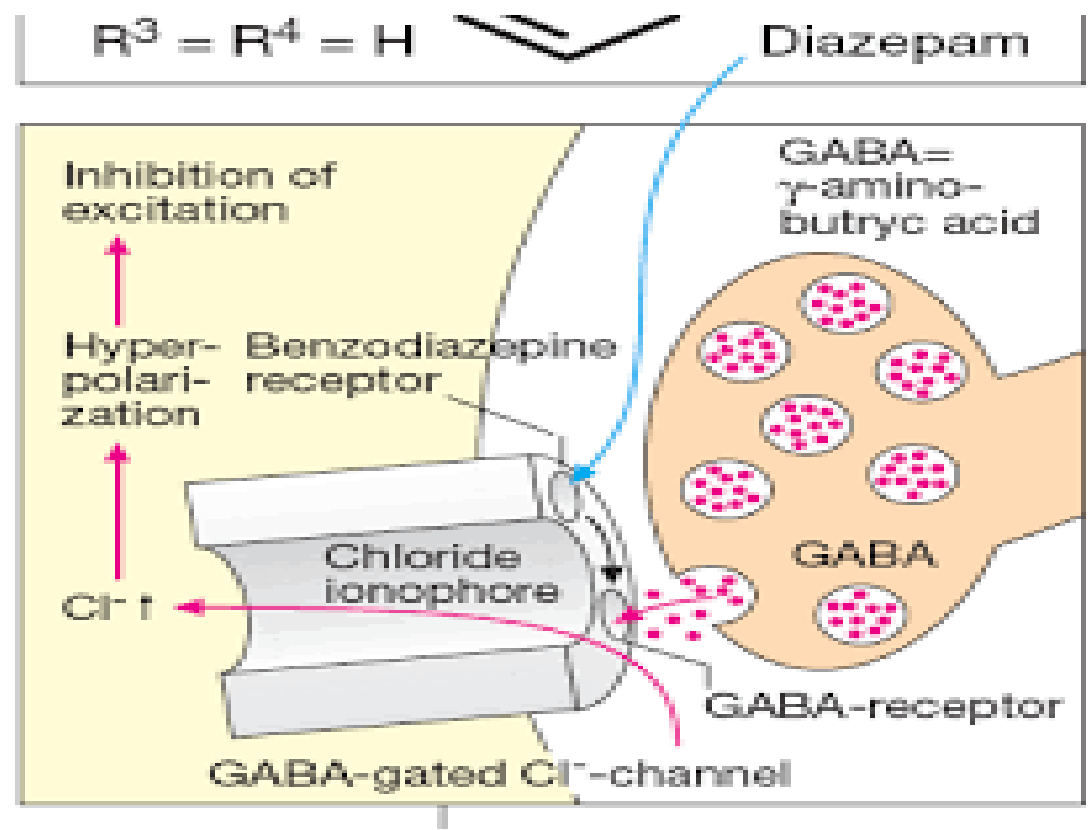

Fonte: Lüllmann, Color Atlas of Pharmacology, 2000.

\subsection{Transtorno do pânico}

O transtorno do pânico está classificado dentro do grupo dos transtornos de ansiedade, e seu diagnóstico é essencialmente clínico, devido a isso podem ocorrer alguns equívocos com relação ao diagnóstico e escolha terapêutica. 
Alguns autores classificam o TP em subtipos em sua apresentação sintomática, tipo cardiorrespiratório, somático, cognitivo. Em que momento do dia ocorrem os ataques, sendo eles diurnos ou noturnos. Idade em que se iniciam, sendo precoce ou tardio e etc. Apesar disso, ainda não existe um material conciso com relação a definição desses subtipos, provavelmente pela semelhança no ponto de vista terapêutico (SALUM et al., 2009).

Há algumas maneiras utilizadas para que seja feita a diferenciação entre ataques de pânicos e outros tipos de ansiedade, há uma série de circunstâncias clínicas e psiquiátricas incluindo ataques de pânico que se assemelham. Portanto, métodos clínicos usados para avaliar são: ataques secundários a condições clínicas, como por exemplo hipertireoidismo; uso ou síndrome de abstinência de substâncias como cocaína, nicotina; transtorno de ansiedade entre outros tipos de transtornos (SALUM et al., 2009).

\subsection{Agorafobia como consequência ao TP}

O tratamento precoce é incentivado ao transtorno de pânico a fim de reduzir o sofrimento e prejuízos associados ao distúrbio e ainda fazer a prevenção em relação ao surgimento de comorbidades e outras complicações como problemas sociais inerentes ao transtorno. Além disso, o tratamento antecipado reduz gastos indiretos com consultas e medicações relativamente desnecessárias em decorrência de um tratamento incongruente com o real problema do paciente (MANFRO et al., 2009).

A diminuição das complicações e prevenção de novas crises propriamente ditas, como a agorafobia, ansiedade antecipatória e a evitação fóbica, são pontos cruciais no tratamento do TP. Deve-se ter muita atenção com relação a comorbidades como os transtornos de humor e também o abuso de substâncias, sejam elas prescritas para o próprio tratamento do transtorno, como os benzodiazepínicos, ou substâncias recreativas como o álcool (MANFRO et al., 2009). 


\section{ANTIDEPRESSIVOS COMO ALTERNATIVA AO TRATAMENTO}

Os antidepressivos são bastante utilizados como alternativa medicamentosa, por agirem propiciando resultados rápidos. É uma opção menos agressiva que os barbitúricos, que por sua vez possuem efeitos adversos bastante graves e baixo índice terapêutico, e causa menos dependência do que os benzodiazepínicos que também podem causar síndrome de abstinência para aqueles que fazem o uso abusivo da substância ou a retirada abrupta da medicação. (DOURADO et al., 2018) Os benzodiazepínicos ainda podem ocasionar efeitos como sedação e comprometimento psicomotor quando associado à outras substâncias ilícitas ou ao etanol, que por sua vez são substâncias com uma prevalência de aproximadamente de $10,6 \%$ na vida dos adolescentes em cidades urbanas do Sudeste (MATTOS et al., 2015).

Estes medicamentos [antidepressivos], atuam no Sistema Nervoso Central aumentando a disponibilidade de alguns neurotransmissores como serotonina, norepinefrina e dopamina na sinapse. Por tanto, é uma classe bastante prescrita por não gerarem dependência na mesma proporção que os benzodiazepínicos e são melhores tolerados pelos pacientes. Dentre as classes dos antidepressivos os mais escolhidos são os Inibidores Seletivos da Recaptação de Serotonina (ISRS), que funciona de forma contrária ao transtorno de ansiedade, que por sua vez ocasiona uma diminuição dos níveis de serotonina no organismo. São ainda escolhidos quando observados sinais depressivos ligados aos distúrbios de ansiedade ou pânico (BRASIL 2017).

Entretanto, em alguns casos, as orientações psicológicas por profissionais da psicologia comportamental já podem ser suficientes sem a necessidade de tratamento farmacológico, ou sendo utilizado apenas em intervalos curtos de tempo préestabelecidos. Tudo isso a depender do estado do paciente, sua doença e grau. Pelo fato de a ansiedade estar diretamente ligada à depressão, os antidepressivos são prescritos com grande frequência (ASSOCIAÇÃO BRASILEIRA DE PSIQUIATRIA 20I3; FIORELLI et al., 2017). 
Figura 2 - Bloqueio da recaptação de serotonina

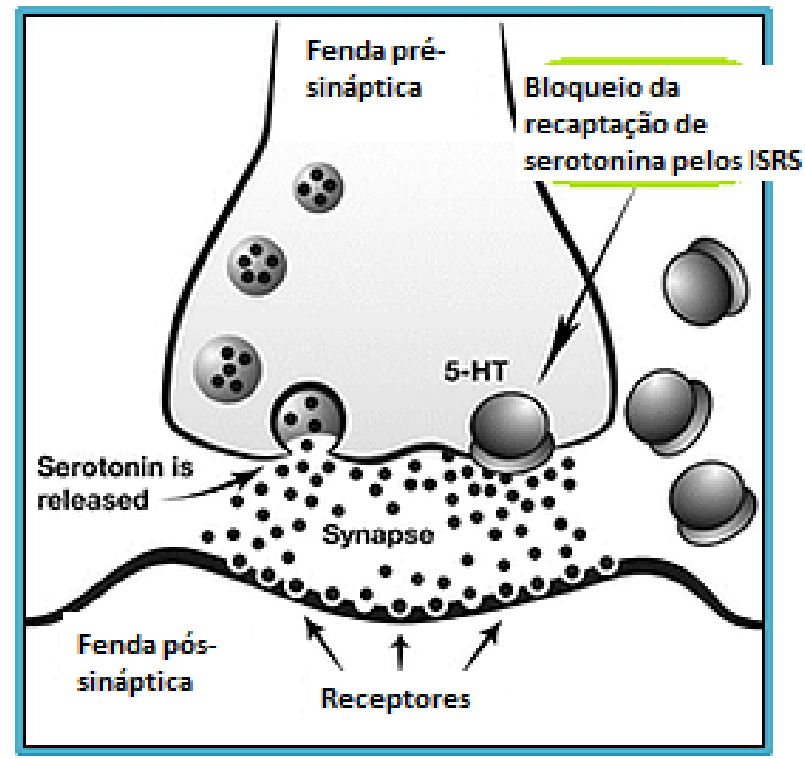

fonte: Adaptado de Lattimore 2005.

\section{PREVENÇÃO}

$\mathrm{Na}$ maior parte dos casos, o TP tem seu prognóstico como um distúrbio crônico com recaídas frequentes, usualmente associadas a momentos de picos de estresse ao longo da vida, possuindo também fases de remissão, seja ela parcial ou completa dos sintomas. Diversos estudos demonstraram que a retirada de medicações pode resultar em recaídas em uma boa parcela de pacientes, aproximadamente 25-50\%, em 6 meses. O $\mathrm{TP}$, sem dúvidas é um transtorno de curso crônico para uma enorme gama de pacientes. Em suma, os ISRS, ADT’s, IRSN e Benzodiazepínicos estão correlacionados à síndrome de retirada (sendo a pior delas com os benzodiazepínicos), sendo até mesmo um promotor de recaída do transtorno de pânico, favorecendo a cronicidade (SALUM et al., 2009).

Por conta da cronicidade dos distúrbios mentais, os profissionais de saúde buscam estudar estratégias que visam a prevenção. Alguns estudos recentes se 
depararam com resultados promissores, utilizando protocolos terapêuticos em indivíduos com sintomas subclínicos do distúrbio. Ainda, sabe-se que fatores de risco para transtornos de ansiedade na idade adulta é o aparecimento de traços de ansiedade na infância e adolescência. Portanto, estudos futuros parecem ter o foco em estudar mecanismos de prevenção em filhos de pais que sofrem ou sofreram algum tipo de transtorno de ansiedade precocemente (SALUM et al., 2009).

\section{CONCLUSÃO}

Por fim, pode-se concluir que o transtorno de ansiedade ou sua vertente mais física, que é o transtorno de pânico, são doenças que em sua maioria se manifesta de forma inesperada em momentos imprevisíveis após traumas ou situações de grande estresse. Especialmente na infância e adolescência é necessário haver prevenção quanto ao surgimento de sintomas, que podem levar à grande cronicidade que levam o indivíduo a administrar medicamentos que podem ser de grande ajuda em um curto período de tempo, mas que rapidamente desenvolvem tolerância, o que transforma a remissão e recaídas em um novo ciclo.

Terapias comportamentais desde o início da adolescência, somados a bons hábitos alimentares e bons convívios, cautela com relação ao uso de substâncias psicotrópicas, sejam elas medicamentos lícitos ou substâncias ilegais, que estão de fato presente no cotidiano do jovem atualmente. $O$ farmacêutico tem um papel imprescindível trazendo alertas que muitas vezes passam desapercebidos por consultas até mesmo em relação a conteúdos básicos e de extrema importância, como por exemplo interações que não podem ocorrer com determinados fármacos, vide benzodiazepínicos e etanol.

Além disso, problemas são inerentes à vida, mas saber como lidar com eles de forma equilibrada e justa, seja consigo mesmo ou com outros, sem causar danos em si mesmo e nem em outrem pode ser o melhor caminho para uma boa saúde mental. 


\section{REFERÊNCIAS BIBLIOGRÁFICAS}

DEMENECH, Lauro Miranda. Under pressure: non-medical use of prescription drugs among undergraduated students, março 2020. Disponível em <scielo.br/j/jbpsiq/a/TQkDSWWB9mB ${ }_{3}$ GXHQ6wSqH9f/?format=html\&lang=en> Acesso em: i7 de setembro de 2021.

FERNANDES, Dione Rodrigues. Uma abordagem ao uso indiscriminado de medicamentos benzodiazepínicos, junho 2018. Disponível em <faema.edu.br/revistas/index.php/Revista-FAEMA/article/view/rcf.vgiedesp.59I〉 Acesso em: i7 de setembro de 2021.

HORTA, Bernardo Lessa. Prevalência e condições associadas ao uso de drogas ilícitas na vida: Pesquisa Nacional de Saúde do Escolar 2015, março 2017. Disponível em < scielo.br/j/rbepid/a/8bBs78WpZKvtcQR4sTpKfpQ/?lang=pt\&format=pdf $>\quad$ Acesso em: I7 de setembro de 202I.

MANFRO, Gisele. Transtornos de ansiedade, dezembro 200o. Disponível em <scielo.br/j/rbp/a/dz9nS7gtB9pZFY6rkh48CLt/?lang=pt> Acesso em: i7 de setembro de 2021.

MAIA, Carlos Renato Moreira. Psicofármacos para o tratamento de transtornos de ansiedade em crianças e adolescentes: uma revisão sistemática, março 2007. Disponível em 〈scielo.br/j/rbp/a/9nVp7HJYGQrSdn73vjB4FLB/?lang=pt> Acesso em: i7 de setembro de 202I. 
NALOTO, Daniele Cristina Comino. Prescrição de benzodiazepínicos para adultos e idosos de um ambulatório de saúde mental, abril 2or6. Disponível em <scielo.br/j/csc/a/C5mWSnzJ68qZ5hqtpJhvpDn/?lang=pt> Acesso em: i7 de setembro de 202I.

SANTOS, Deise Kelly Lopes. Transtorno de ansiedade na juventude e o uso abusivo de benzodiazepínicos, novembro 2019. Disponível em <http://repositorio.faema.edu.br/bitstream/I23456789/2473/I/TCC_DEISE\%2oKELY\% 2oLOPES\%2oSANTOS_CORRIGIDO_assinado.pdf> Acesso em: I7 de setembro de 2021.

SALUM, Giovanni Abrahão. Transtorno do pânico, fevereiro 2009. Disponível em <scielo.br/j/rprs/a/VgdKjMfjhGfGcFTdBgYCq6G/?lang=pt\&format=pdf $>$ Acesso em: I7 de setembro de 202I. 